\title{
Sexualidad y cáncer: toxidicidad y tratamientos de soporte
}

\author{
E. López Ramírez' , E. González Flores²
}

\section{Introducción}

La sexualidad es un universo simbólico que empieza en la fisiología, atraviesa los tupidos campos de la religión, la psicología, la economía, la política para llegar a la ética ${ }^{1}$.

Es una materia muy personal y significa diferentes cosas para diferentes personas. Las actitudes y los comportamientos sexuales pueden variar enormemente de persona a persona y además cambiar según circunstancias. Para este trabajo nos basaremos en una concepción occidentalizada de la sexualidad.

Las necesidades y sentimientos relacionados con la sexualidad son una preocupación presente en los pacientes y un desafío para los profesionales, al tratarse de un tema íntimo, de delicado manejo, pero real y vigente, que requiere de determinadas habilidades de exploración y tratamiento ${ }^{2}$.

Según el Sistema Clasificatorio Integrado Tipológico-Funcional de los problemas sexuales propuesto por Carrobles J.A y Sanz A. ${ }^{3}$, los pacientes oncológicos presentan fundamentalmente disfunciones sexuales (aunque pueden tener también otros). Además estos se matizan según la temporalidad, la situación, la intensidad y la etiología.

\section{Frecuencia y tipos de disfunción sexual en las personas con cáncer}

En las tentativas para evaluar la prevalencia de disfunción sexual atribuible a la enfermedad y/o al tratamiento oncológico, se han encontrado valores que oscilan entre el 35 y el $85 \%$ en hombres y mujeres ${ }^{4}$.

Aproximadamente el $50 \%$ de las mujeres que han tenido cáncer de la mama sufren de disfunciones sexuales prolongadas $^{5,6}$ al igual que una proporción similar de mujeres que han tenido cáncer ginecológico ${ }^{7}$. Según Potosky $\mathrm{AL}$ et al ${ }^{8}$, a los cinco años de seguimiento, la disfunción eréctil tras una

Servicio de Oncología Radioterápica

2 Servicio de Oncología Médica

Hospital Universitario Virgen de las Nieves

Granada prostatectomía radical es de un $79.3 \%$ y de un $63.5 \%$ después de la radioterapia de haz externo. La disfunción es menor cuando se emplea la braquiterapia. Hay que tener en cuenta que pueden existir sesgos de selección de los pacientes para los distintos tratamientos, como que sean intervenidos con prostatectomía y preservación de nervios los pacientes con buena función sexual previa, dejando para tratamiento con radioterapia pacientes con mal estado general. Además los pacientes pueden mejorar un año después de la cirugía y sin embargo empeorar después de dos años de la radioterapia.

Los problemas sexuales más comunes para las personas con cáncer son la falta de apetito sexual en el hombre y en la mujer, disfunción eréctil en el hombre y dispareunia en la mujer 6 .

\section{Salud sexual y rehabilitación sexual}

Implantar programas de rehabilitación sexual, prácticos, económicos y eficaces es un desafío constante.

Definimos la rehabilitación sexual como la curación del impacto emocional del cáncer sobre la imagen corporal, la satisfacción de las relaciones y la capacidad reproducti$\mathrm{va}^{9}$.

Muy raras veces síntomas relacionados con el tumor maligno por sí mismo interfieren con la función sexual (anemia, anorexia, dolor), más bien los tratamientos (cirugía, radioterapia o quimioterapia) o las alteraciones psicológicas que producen ambos (creencias, impactos emocional, motivaciones, afrontamientos), son los responsables y pueden afectar una o más fases del ciclo de respuesta sexual (sobre todo deseo, excitación y orgasmo) ${ }^{2}$.

La mayor parte de los cánceres se producen con más frecuencia a medida que aumenta la edad y muchos pacientes tienen antecedentes médicos que podrían ya alterar la respuesta sexual ${ }^{10}$. Entre los medicamentos que pueden afectar en mayor medida la función sexual, se encuentran: los quimioterápicos, las hormonas (testosterona y estrógenos), algunos antibióticos y antiparasitarios, los anticolinégicos y antisecretorios $\mathrm{H} 2$, los antidepresivos tricíclicos, neurolépticos, ansiolíticos e hipnóticos, diuréticos y drogas antihipertensivas, etcétera. Otros productos como los opioides, el alcohol, las anfetaminas, la cocaína y los alucinógenos, pueden afectar la respuesta sexual de diferentes modos ${ }^{11}$. Enfermedades tan frecuentes como la hipertensión y la diabetes causan 
problemas de erección y eyaculación, el hipotiroidismo produce desinterés sexual y al contrario el hipertirioidismo, las lesiones del lóbulo frontal causan desinhibición...11

Los profesionales debemos conocer los tipos de problemas que pueden surgir después de determinados tratamientos oncológicos y modificar el asesoramiento de los pacientes con cáncer.

\section{Ciclo psico-fisiológico de la respuesta sexual humana}

Para enfocar los problemas sexuales en pacientes con cáncer se recurre comúnmente al ciclo de la respuesta sexual humana $^{12}$, que actualmente consta de 6 fases dos subjetivas y cuatro objetivas ${ }^{3}$.

Cada fase corresponde a la siguiente definición:

1) El deseo sexual o líbido es el nombre que recibe el interés por el sexo. Puede alterarse por circunstancias como el ciclo menstrual, el embarazo, la lactancia, la menopausia, aspectos psicológicos, etc.

2) La excitación es el despertar de los sentimientos sexuales. Pueden producirse por mirar a alguien, el tacto, una fantasía sexual, o contacto con zonas genitales o erógenas. Puede conducir o no al orgasmo.

3) La meseta que es la fase en la que el cuerpo mantiene un elevado estado de excitación. El cuerpo es muy sensible en esta fase.

4) El orgasmo o clímax sexual y los sentimientos de intensa sensación que ocurren en las áreas del cuerpo dan origen a contracciones rítmicas. Las mujeres pueden sentir contracciones en el útero y los hombres eyaculan (excepto que tengan una vasectomía hecha).

5) La resolución que es la fase que sigue a la excitación y el orgasmo. Los cambios corporales vuelven a la normalidad. Los hombres no pueden normalmente excitarse otra vez durante un período (refractario). Algunas mujeres pueden ser capaces de excitarse y tener un orgasmo nuevamente. Con la edad este período refractario aumenta.

6) La satisfacción sexual de naturaleza estrictamente psicológica o subjetiva, evaluada en términos del grado de satisfacción, positiva o negativa, experimentado por el sujeto después de la actividad sexual.

Cada una de estas fases está caracterizada por experiencias subjetivas, eventos objetivos que pueden ser medidos y por sistemas fisiológicos que necesitan estar intactos para que estos eventos ocurran. La excitación, la meseta y el orgasmo sólo suceden si el cuerpo tiene un buen aporte de sangre, si el sistema nervioso central y periférico están intactos, si existe un buen soporte muscular y si el balance hormonal del cuerpo es correcto ${ }^{13}$.

\section{Asesoramiento sexual}

La mayoría de los hombres y mujeres nunca son interrogados sobre sus problemas sexuales por parte del equipo médico. Se aduce la presión del tiempo pero puede ocultar otros problemas como vergüenza o que no tendrán soluciones que ofrecer. Algunos trabajos demuestran que los médicos subvaloran la sexualidad de sus pacientes ${ }^{14}$.

Lo ideal sería una entrevista pretratamiento para discutir con el paciente las dificultades sexuales que pueden apare- cer y reducir así su ansiedad. Preguntar al paciente durante el tratamiento y seguimientos posteriores.

Se estima que entre un 80 y un $90 \%$ de los pacientes se benefician de un breve consejo sexual y no necesitan de una consulta especializada ${ }^{15}$. Existen modelos generales de intervención terapéutica como el Modelo P-LI-SS-IT (Permision, Limited information, Specific suggestions, Intesive Therapy) ${ }^{16} \mathrm{O}$ EPITEX (Educación, Permiso, Indicaciones específicas, Terapia Sexual) ${ }^{3}$. Los indicadores para derivación a terapia sexual son: problema sexual previo al cáncer, conflictos matrimoniales severos, disfunción que no ha respondido al asesoramiento sexual breve o un problema sexual que es sólo una faceta de una mala adaptación psicológica.

Es preciso proporcionarle al paciente información de su anatomía, ciclo de respuesta sexual, impacto del tratamiento sobre la función sexual.

\section{Evaluación de la función sexual en pacientes con cáncer}

Se debe realizar una pregunta general sobre el sexo en la historia clínica inicial o en las visitas de seguimiento. Ej: Un aspecto de la salud de toda persona es la salud sexual ¿Cómo ha afectado el diagnóstico de cáncer a su vida sexual?

En un ámbito multidisciplinario puede ser útil designar a un médico, enfermera o asistente social entrenados como experto en rehabilitación sexual. Incluso en los principales centros oncológicos es raro encontrar un especialista en salud sexual.

Se puede decir que la evaluación de éstos incluye un interrogatorio exhaustivo (entrevistas privadas a solas y con la pareja estable y/o cuestionarios estandarizados), un exámen físico completo, exámenes complementarios (análisis sanguíneo completo, determinación de niveles séricos hormonales y técnicas de imágen), evaluaciones neurológica, vascular y psicológica detalladas.

\section{Soluciones a los problemas sexuales causados por el cáncer y sus tratamientos}

Hay cuatro vías principales en las que el cáncer o sus tratamientos pueden afectar su sexualidad:

- Problemas físicos para dar y recibir placer sexual

- Alteración de la imagen corporal

- Aparición de sentimientos como miedo, tristeza, angustia..

- Modificación de roles y relaciones.

Las relaciones entre estas cuatro áreas son importantes. $\mathrm{Si}$ hay un problema en alguna de ellas, esto puede tener un impacto en las otras.

\section{Desajuste en el deseo}

Es importante que el paciente comunique a su pareja que ha perdido interés por la actividad sexual debido al estigma, la mutilación o los obstáculos físicos, ya que esto evita sentimientos de rechazo. Se deben sugerir alternativas de proximidad y afecto como caricias, autoerotismo y otras técnicas de focalización sensitiva ${ }^{12,17}$.

Si se siente cansancio se debe hacer el amor de un modo diferente: posiciones menos cansadas donde el peso sea 


\section{E. López Ramírez y E. González Flores}

bien soportado, se puede recurrir a un contacto sexual más rápido y/o mantener la relación por la mañana. Siempre hay que hablar y consensuar con la pareja.

\section{Disminución del impulso sexual}

Si existen problemas hormonales por menopausia prematura se puede utilizar la terapia hormonal sustitutiva con estrógenos y quizás con testosterona (valorando individualmente cada caso) $)^{18,19,20 .}$

El sildenafilo y fármacos similares pueden usarse para aumentar el impulso sexual en las mujeres. Aumentan la lubricación vaginal y disminuyen el dolor durante el coito, aseguran más excitación y facilitan el alcance del orgasmo.

Muchas veces existen problemas educacionales y hay que recordarle a la paciente que debe "darse permiso para sentir placer".

En varones con cáncer de testículo y bajos niveles de testosterona se puede utilizar tratamiento de reemplazo con testosterona que alivian, con frecuencia, la pérdida de deseo sexual o la disfunción eréctil.

\section{Dispareunia}

La cirugía, la quimioterapia, la terapia hormonal, la radioterapia o algunos medicamentes (Ej: antihistamínicos) puede causar cambios que producen sequedad vaginal, estrechez, úlceras o infecciones. Esto ocasiona dolor durante el coito.

Una experiencia de dolor puede desencadenar temor al mismo y conducir a tensión. Esta tensión distrae a la persona de alcanzar la excitación, detiene la lubricación y causa nuevamente dolor.

Es importante mostrarles con un espejo sus genitales para que sean capaces de conocer su vulva, su introito y reconozcan zonas dolorosas o no. Indicarles la posición del punto Grafenberg (punto G), aunque puede ser de difícil acceso en algunas mujeres ${ }^{21}$.

Es importante comentarlo con el compañero/a para explorar nuevas posiciones para hacer el amor. El coito lateral puede disminuir el peso sobre heridas o cicatrices. La persona que experimenta dolor debe controlar la profundidad y la velocidad de la penetración. Puede ser también útil aproximar la eyaculación a la penetración lo que acorta la duración del acto sexual.

Pueden beneficiarse a menudo aprendiendo ejercicios para relajar los músculos que rodean la entrada de la vagina (Ejercicios de Kegel). Esto no es para estirar físicamente la vagina sino para proporcionar la sensación de que puede relajar la entrada de la vagina y permitir la penetración del pene sin experimentar dolor 22,23 .

También puede ser beneficioso planear el contacto sexual y tomar analgésicos previamente. Las almohadas y cojines pueden utilizarse para estar más cómodo.

También es conveniente comentarlo con el médico para que realice una exploración y diagnostique el problema proponiendo soluciones.

Si existe sequedad vaginal pueden utilizarse cremas y geles que se aplican directamente en la vagina, contienen pequeña cantidad de estrógenos naturales y semisintéticos que tienen un efecto poco duradero y que no influyen en el resto del cuerpo.
Los lubricantes con agua pueden aumentar la humedad haciendo más fácil la penetración. Algunas mujeres prefieren usar glicerina que es más barata y menos embarazosa porque se puede usar para muchas cosas.

Si el problema es la estrechez vaginal (frecuente después de la radioterapia y de la cirugía), se recomienda usar dilatadores vaginales que varían en tamaño y se aplica uno mismo, incluso como parte del juego sexual. Previenen que las paredes de la vagina se unan y se usan junto a lubricantes. También se puede recurrir a tampones o a mantener relaciones sexuales muy frecuentes.

Cuando existen úlceras vaginales producidas por radioterapia, estas sangran levemente y tardan semanas o meses en cicatrizar.

Las infecciones vaginales, tras radioterapia o quimioterapia, debido a que existen cambios en la acidez de la vagina lo que permite a los microorganismos crecer. Si se aprecia una secreción cremosa blanquecina o picor que empeora con el rascado se debe consultar pues se pueden tener hongos o aftas. Si se han mantenido relaciones el compañero sexual también debe tratarse con antifúngicos y/o antivíricos.

Si no se tienen estas molestias durante los tratamientos, las relaciones sexuales son seguras. Se deben usar métodos anticonceptivos cuando sea necesario.

\section{Eyaculación retrógrada}

Los pacientes que no se han sometido a Linfadenectomía Abdomino Retroperitoneal (LARP) y tienen este problema parcialmente (neurotoxicidad por quimioterapia o radioterapia) pueden ser tratados con agentes alfa-simpáticomiméticos o estimulación eléctrica/vibratoria para inducir la eyaculación anterógrada de semen ${ }^{24,25,26}$.

\section{Disfunción eréctil}

Una erección incompleta puede ser efectiva para mantener una relación. La posición mejor para ello es con la pareja encima guiando el pene al interior.

Si el paciente ha tenido una intervención que ha dañado los nervios necesarios para la erección, no necesariamente tiene que terminar con su actividad sexual. No se requiere un pene firme para proporcionar placer al compañero/a. Se pueden introducir o aumentar prácticas de sexo oral, caricias, masturbación o vibradores para aumentar la excitación de ambos.

Existen varios fármacos eficaces:

1) Sildenafilo: Se toma una hora antes de la relación sexual y la estimulación directa posterior origina una erección. No se recomiendan en personas con problemas de corazón o que está tomando ciertas drogas como nitratos. Pueden causar efectos secundarios como dolor de cabeza, mareo, cambios visuales y palpitaciones. A veces la erección puede durar un par de horas o más y dañar los tejidos del pene (priapis$\mathrm{mo})^{27}$.

2) Vardenafilo: Tarda en funcionar 25-60 minutos. El efecto secundario más común es el dolor de cabeza y el enrojecimiento de la cara.

3) Tadalafilo: Se puede tomar 36 horas antes de la relación. 
4) Hidroclorhidrato de Apomorfina: Se disuelve bajo la lengua. Proporciona una erección en 10-20 minutos. Puede causar dolor de cabeza, nauseas y mareo.

5) Prostaglandina El inyectada directamente en el pene. El fármaco restringe el aporte sanguíneo y atrapa la sangre en el pene. Hace falta práctica para usar la dosis correcta. Un efecto secundario posible es el de administrar demasiado fármaco y que la erección permanezca durante demasiado tiempo dañando los tejidos. Algunos pacientes que usan este método dicen que la cabeza del pene no está tan firme como el tronco. Se recomiendan una-dos veces a la semana lo que no es suficiente para algunos hombres.

Las bombas de vacío consisten en un tubo hueco en el que se introduce el pene. La bomba tiene un mango que succiona sangre hacia el pene creando un vacío. La sangre queda capturada en el pene por un anillo de goma situado en la base del pene y provoca una erección. Se puede mantener 30 minutos. Una vez que se ha finalizado se retira el anillo y la sangre fluye normalmente otra vez. No requiere insertar nada en el pene pero requiere un par de semanas para acostumbrarse a su uso. Ayuda a las personas que no pueden tomar otras medicinas. La pareja puede notar el pene algo más frío que en otras ocasiones ya que la sangre no se está moviendo. Otro aspecto importante es tener el anillo puesto sólo durante media hora cada vez. Se puede usar las veces que uno quiera pero dejando 30 minutos entre cada uso para que la sangre circule adecuadamente en el pene y evitar la isquemia peneana. La parte proximal del pene no queda tan firme y precisa conducirlo para la penetración además puede comprimirse la uretra y tener un orgasmo se$\mathrm{CO}^{28,29}$

El implante de una prótesis peneana es potencialmente irreversible. Representa una solución permanente de la disfunción eréctil para más del $95 \%$ de los casos. Es el tratamiento óptimo para la disfunción eréctil irreversible en un paciente con buena expectativa de vida. Como muchos pacientes recuperan de forma gradual la rigidez de la erección después de las operaciones conservadoras de nervios, el momento óptimo para el implante oscila entre los 6-12 meses después de la cirugía oncológica. Las prótesis inflables son las que más se utilizan en la actualidad. El reservorio líquido se puede poner penescrotal o extraperitoneal. La intervención dura menos de 2 horas y 1 semana de hospitalización ${ }^{30}$.

Insistir en que el hombre no olvide que la mujer precisa de estímulos para excitarse y lubricarse.

\section{Problemas de la imagen corporal}

La imagen corporal es el dibujo mental que tenemos de nuestra propia apariencia. Cuando hay un cambio en la imagen corporal de modo rápido y dramático la persona se siente anormal, sexualmente menos atractiva.

Existen cambios que pueden ser ocultados bajo la ropa como la colostomía, urostomía o la mastectomía. Es frecuente que la persona reaccione intentando pasar como "normal" para el observador casual, pero esto es imposible para la pareja sexual. Ocultan el cambio, evitan mirarlo y lo ocultan de los demás. Esta evitación puede hacer que la persona se sienta cada vez más ansiosa respecto a que alguien descubra el cambio.
En estos casos se puede intentar tener relaciones sexuales con la ropa interior o parcialmente vestido antes que completamente desnudo. Cambiar el nivel de la luz puede ayudar a ir adquiriendo confianza sobre como ve su propio cuerpo. Puede ayudar el tumbarse sobre el lado para prevenir la presión en las cicatrices o estomas. Recomendarle verse frente al espejo o tocarse la herida

Existen alterariones visibles como la pérdida de la nariz, cicatrices faciales, alopecia, palidez, alteraciones cutáneas y cambios de peso. En estos casos debe emplearse la reestructuración cognitiva y técnicas de mejora de autoestima.

\section{Adaptación psicológica a los sentimientos}

Existen factores de riesgo previo a la enfermedad como una valoración pesimista de la vida, antecedentes de trastornos del estado de ánimo o de ansiedad, o falta de recursos de salud, financieros o sociales.

Los pacientes jóvenes también tienen mayores probabilidades de sufrir distrés psicológico porque sufren un deterioro mayor de los objetivos de su vida como establecer una relación comprometida, tener hijos y progresar en su carrera ${ }^{31,32}$.

La depresión, ansiedad, miedos..., hacen que sea improbable la estimulación cuando se piensa en el sexo. Algunas personas sienten un aumento en la excitación sexual lo que les ocasiona culpabilidad.

El diagnostico de cáncer no produce alteración matrimonial en parejas felices pero puede exacerbar los conflictos de parejas que ya tenían problemas.

Se deben compartir los sentimientos con la pareja, con los especialistas, con un miembro de la familia que le escuche $y$ no le juzgue.

\section{Alteración de roles y relaciones}

El cáncer cambia el rol de la persona en la familia ya que puede perderse la energía física para hacer todos los trabajos que se hacían antes. Por ejemplo, la expresión del afecto no sexual disminuye mucho en las parejas en las que la mujer tiene cáncer. Cuando el hombre es el que lo padece, la pérdida de la función sexual y de la capacidad de ingreso económico son dos elementos que, frecuentemente, se consideran como pérdida simbólica de masculinidad 33,34

Si se está buscando pareja, puede ser difícil decidir que contar sobre la enfermedad y cuando.

Si se es homosexual esto puede suponer más flexibilidad y variedad en la vida sexual. Esto puede ser de ayuda cuando se esta intentando adaptarse a los cambios del cáncer y sus tratamientos. Si el sexo es muy importante en su vida, la pérdida de la función sexual puede ser difícil de aceptar. La fertilidad también es importante para los homosexuales.

\section{Embarazo, contracepción e infertilidad}

Discutir siempre los pros y contras de continuar con el embarazo si este se ha producido. Se recomienda no utilizar la píldora como método anticonceptivo ya que los vómitos y la diarrea la hacen menos efectiva, son mejores los métodos de barrera.

En mujeres jóvenes, la infertilidad no sólo es fuente de angustia, sino que también algunas pueden llegar a evitar las relaciones sexuales por temor de que un embarazo provo- 


\section{E. López Ramírez y E. González Flores}

que la recidiva del cáncer, especialmente después de un cáncer de mama sensible a hormonas. La evidencia actual indica, sin embargo, que el embarazo en el momento del diagnóstico de cáncer de mama en estadio precoz o después del tratamiento local exitoso, no afecta la sobrevida libre de enfermedad en mujeres con ganglios negativos ${ }^{35,36}$.

La cirugía, la radioterapia, la quimioterapia o la hormonoterapia pueden causar infertilidad.

No existe una forma mejor o peor de reaccionar pero se debe dar información y discutir las opciones (incluyendo a la pareja) con el equipo médico, psicólogos y Unidad de Reproducción. Se aconseja realizar seminogramas y criopreservación.

En Bélgica se ha conseguido el nacimiento de una niña tras un trasplante (autotrasplante ortotópico en la cavidad pelviana) de un fragmento de ovarios tomado de la propia paciente y luego criopreservados antes del inicio de la quimioterapia por un linfoma de Hodgkin y siete años después de haber finalizado los tratamientos.

\section{Impacto del tratamiento oncológico sobre la sexualidad de pacientes pediátricos y terminales}

Existen pocos datos. Dos encuestas realizadas en jóvenes supervivientes de cáncer en la infancia revelaron que las tasas de matrimonio eran menores que en la población gene$\mathrm{ral}^{37,38}$.

Los tratamientos oncológicos pueden causar trastornos de la función gonadal y cognoscitiva ${ }^{39}, 40$, pero sólo un grupo ha investigado aspectos sexuales. En este trabajo todos los pacientes estaban por debajo de la normalidad en términos de actitudes, intereses y experiencias sexuales ${ }^{41}$.

Es importante identificar, reconocer y estimular conductas sexuales alternativas, que sin representar maltrato, vergüenza o humillación, ofrezcan posibilidades placenteras, como la cercanía física no genital y variadas demostraciones de afecto. Sin duda, una prolongada mirada, una tierna caricia, una auténtica sonrisa de amor, pueden compensar (incluso en momentos postreros) una ejecución sexual en otros tiempos deseada, llevar a la satisfacción de las necesidades de este paciente en esos momentos ${ }^{42}$. Hay que concebir aquí a la sexualidad en su más abierta y flexible expresión.

\section{Bibliografía}

1. Marina JA. El rompecabezas de la sexualidad. Ed Anagrama. Barcelona. 2002.

2. Grau Abalo JA. La sexualidad en pacientes con cáncer: algunas consideraciones sobre su evaluación y tratamiento. Rev Cubana Oncol 1999;15(1):49-65

3. Carrobles JA, Sanz A. Clasificación de los problemas sexuales. En: Carrobles JA, Sanz A. Terapia Sexual. Ed. Fundación Universidad-Empresa. Madrid, 1998: 27-55.

4. Schavelzon J. Desadaptación afectiva, sexualidad y cáncer. En: Schavelzon J, ed. Psique. Buenos Aires: Científica Interamericana, 1992:258-74.

5. Ganz PA, Rowland JH, Desmond K, et al. Life after breast can- cer: understanding women's health-related quality of life and sexual functioning. J Clin Oncol 16 (2): 501-14, 1998.

6. Schover LR, Montague DK, Lakin MM. Sexual problems. In: DeVita VT Jr, Hellman S, Rosenberg SA, eds.: Cancer: Principles and Practice of Oncology. 5th ed. Philadelphia, Pa: LippincottRaven Publishers, 1997, pp 2857-2872.

7. Andersen BL. Quality of life for women with gynecologic cancer. Curr Opin Obstet Gynecol 7 (1): 69-76, 1995.

8. Potosky AL, Davis WW, Hoffman RM et al. Five year outcomes after prostatectomy or radiotherapy for prostate cancer: the prostate cancer outcomes study. J Natl Cancer Inst 96 (18): 1358-67, 2004.

9. Schover LR, Schain WS, Montague DK. Sexual problems of patients with cancer. In: Perez C.A., Brady L. W. Principles and practice of radiation oncology. Philadelphia: J. B. Lippincott company $2^{\mathrm{a}}$ ed. 1992: 2206-2225.

10. Hawton K. Terapia Sexual. Ed. Doyma Barcelona. 1988.

11. Labrador FJ. Causas fisiológicas de las disfunciones sexuales. En: Labrador FJ. Enciclopedia de la sexualidad. Espasa Calpe Madrid. 2000.

12. Masters WH, Johnson VE. Human sexual response. Ed. Little Brown. Boston. 1970.

13. Oliven JF. Clinical Sexuality. Ed. Fhiladelphia J.B., 1974: 175186.

14. Opjordsmoem S, Waehre H, Aass N. Sexuality in patients treated for penile cancer: patients experience and doctors judgement. Br J Urol 1994;73(5):554-60.

15. Schover LR, Schain WS, Montague DK. Psychologic aspects of patients with cancer: Sexual problems in patients with cancer. En: Clinical oncology: A multidisciplinary approach, 2 ed. New York: American Cancer Society, 1989:2220-5.

16. Annon JS. Behavioral treatment of sexual problems. Ed. Harper and Row, New York, 1976: 45-47.

17. Kaplan HS. The new sex therapy: Active treatment of sexual dysfunctions. New York, NY: Brunner/Mazel, 1974.

18. Kaplan HS, Owet T. The female androgen deficiency syndrome. J Sex Marital Ther 1993; 19: 3.

19. Inskip PD. Pelvic radiotherapy, sex hormones, and breast cancer. Cancer Causes Control 1994; 5: 471.

20. Yasuda M, Kurabayashi T, Yamamoto Y, Fujimaki T, Oda K, Tanaka K. Effect of hormone replacement therapy on bone and lipid metabolism in women oophorectomized for the treatment of gynecologic malignancies. Int J Gynecol Obstet 1994; 47: 151.

21. De la Hermosa AR. Los órganos sexuales femeninos. En: Labrador FJ. Enciclopedia de la sexualidad. Espasa Calpe Madrid. 2000

22. Fordney DS. Dyspareunia and vaginismus. Clin Obstet Gynecol $1978 ; 21: 205$

23. Christensen LL, Diurhuus JC, Lewis MT, et al. MRI of voluntary pelvic floor contractions in healthy female volunteers. Int Urogynecol J 1995; 6:138.

24. Donohue JP, Foster RS, Rowland RG, et al. Nerve-sparing retroperitoneal lymphadenectomy with preservation of ejaculation. J Urol 1990; 144:287.

25. Jewett MAS, Kong YP, Goldberg SD, et al. Retroperitoneal lymphadenectomy for testis tumor with nerve sparing for ejaculation. J Urol 1988; 139: 1220

26. Recker F, Tscholl R. Monitoring of emission as direct intraoperative control for nerve sparing retroperitoneal lymphadenectomy. J Urol 1993; 150: 1360. 
27. Goldstein I, Lue TF, Padma-Nathan, et al. Oral sildenafil in the treatment of erectile dysfunction. Sildenafil Study Group. N Engl J Med 338(20): 1397-404, 1998.

28. Sidi AA, Becher EF, Zhang G, Lewis JH. Patient acceptance of and satisfaction with an external negative pressure device for impotence. J Urol 1990; 144: 1154.

29. Turner LA, Althof SE, Levine SB, et al. Treating erectile dysfunction with external vacuun devices: impact upon sexual, psychological and maritalfunctioning. J Urol 1990; 144: 79.

30. Montague DK. Penile prostheses: an overview. Urol Clin N Am 1989; 16:7.

31. Caner CS, Pozo-Kaderman C, Harris SD, et al. Optimism versus pessimism predicts the quality of women's adjustment to early stage breast cancer.Cancer 1994; 73: 1213.

32. Ganz PA, Hirii K, Sim M, Schag CAC, Fred C, Plinsky ML. Predicting psychosocial risk in patients with breast cancer. Med Care 1993; 31:419.

33. Schover LR, Evans RB, von Eschenbach AC. Sexual rehabilitation in a cancer center: diagnosis and outcome in 384 consultations. Arch Sex Behav 1987; 16:445.

34. Liss-Levinson WS. Clinical observations on the emotional responses of males to cancer. Psychother Theory Res Pract 1982; 19:325.

35. Dow KH, Harris JR, Roy C. Pregnancy after breast-conserving surgery and radiation therapy for breast cancer. J Nat Cancer Inst 1994; 16:131

36. Von Schoultz E, Johansson H, WilkingN, Rutqvist LE. Influence of prior and subsequent pregnancy on breast cancer prognosis. J Clin Oncol 1995; 13:430.

37. Green DM, Zevon MA, Hall B. Achievement of life goals by adult survivors of modern treatment for childhood cancer. Cancer 1991; 67: 206.

38. Byrne J, Fears TR, Steinhorn SC, et al. Marriage and divorce after chilhood and adolescent cancer. JAMA 1989; 262: 2693.

39. Ochs J, Mulhern R. Long-term sequelae of therapy for chilhood acute lymphoblastic leukaemia. Ballieres' Clin Haematol 1994; 7: 365 .

40. Mustieles C, Muñoz A, Alonso M, et al. Male gonadal function after chemotherapy in survivors of childhood malignancy. Med Ped Oncol 1995; 24: 347.

41. Ropponen P, Aalberg V, Rautonen J, Kalmari H, Siimes MA. Psychosexual development of adolescent males after malignancies in childhood. Act Psychiatr Scand 1990; 82:213.

42. Grau Abalo J.A., Jiménez PJ. La sexualidad en el enfermo de cáncer. En: Gómez Sancho M. Cuidados paliativos e intervención psicosocial en enfermos terminales, 2 ed. Las Palmas de Gran Canaria: ICEPSS, 1988:67-89. 